\title{
Conventional oral systemic chemotherapy for postoperative hepatocellular carcinoma: A systematic review
}

\author{
JIANHONG ZHONG, BANGDE XIANG, LIANG MA and LEQUN LI \\ Department of Hepatobiliary Surgery, Affiliated Tumor Hospital of Guangxi \\ Medical University, Nanning, Guangxi 530021, P.R. China
}

Received October 8, 2012; Accepted June 30, 2014

DOI: $10.3892 / \operatorname{mco} .2014 .337$

\begin{abstract}
The findings of randomized clinical trials (RCTs) regarding the efficacy of adjuvant conventional oral systemic chemotherapy (COSC) for patients with hepatocellular carcinoma (HCC) following curative hepatic resection (HR) are contradictory. Therefore, a systematic review of RCTs is required to evaluate the clinical efficacy of adjuvant COSC. Sources such as Medline, Embase and the Cochrane Library were systematically searched and all the RCTs comparing curative HR alone to $\mathrm{HR}$ plus COSC for HCC were identified. The odds ratios (ORs) and 95\% confidence intervals (CIs) were calculated. No treatment-related mortality was reported by the included RCTs and the adverse effects of COSC were generally mild. However, adjuvant COSC did not achieve a statistically significant improvement in the 1-, 3- and 5-year overall survival $(\mathrm{OR}=1.43$, 95\% CI: 0.58-3.56, $\mathrm{P}=0.44$; $\mathrm{OR}=1.39,95 \% \mathrm{CI}: 0.75-2.55, \mathrm{P}=0.29$; and $\mathrm{OR}=1.20$, 95\% CI: 0.46-3.16, $\mathrm{P}=0.71$, respectively). In addition, adjuvant COSC did not achieve a statistically significant decrease in the incidence of 1-, 3- and 5-year tumor recurrence, with pooled ORs of 0.92 (95\% CI: 0.26-1.35, $\mathrm{P}=0.66)$; 0.82 (95\% CI: 0.66-1.01, P=0.06); and 0.84 (95\% CI: 0.71-1.01, $\mathrm{P}=0.06)$, respectively. Narrative reviews offer no evidence supporting the use of COSC. Adjuvant COSC has provided marginal benefits for HCC patients following curative HR. Considering the efficacy of sorafenib for advanced HCC and the results of this systematic review, no further trials should be performed to assess the efficacy of adjuvant COSC.
\end{abstract}

\section{Introduction}

Hepatocellular carcinoma (HCC) is a malignancy associated with a poor prognosis and has a heterogeneous composition,

Correspondence to: Professor Liang Ma or Professor Lequn Li, Department of Hepatobiliary Surgery, Affiliated Tumor Hospital of Guangxi Medical University, $71 \mathrm{He}$ Di Road, Nanning, Guangxi 530021, P.R. China

E-mail: maliang0123@126.com

E-mail: xitongpingjia@163.com

Key words: hepatocellular carcinoma, oral, systemic chemotherapy, systematic review with multiple variables that vary among different regions. Although several novel treatment options have been investigated and used in the clinical setting, hepatic resection (HR) remains the primary treatment for HCC. However, even after curative resection, recurrence is common and remains the main cause of mortality. The 3 -year tumor recurrence rate was reported to be $>60 \%$ following HR $(1,2)$ and the 5 -year overall survival (OS) rate ranges between 39 and $50 \%(3,4)$. Consequently, adequate and effective adjuvant therapy is essential to improve the OS of HCC patients.

Various types of postoperative therapies, such as transarterial therapy with or without embolization, systematic therapy, interferon, lamivudine, vitamin $\mathrm{A}$ and $\mathrm{K} 2$ analog and adoptive immunotherapy, have been reported for HCC patients following curative treatment. However, interferon is frequently associated with various adverse effects (5) and postoperative transarterial chemoembolization appears to be promising only for HCC patients with a high risk of recurrence (6). Adoptive immunotherapy, although associated with a lower recurrence rate following surgery for $\mathrm{HCC}$, does not appear to increase OS (7), whereas the manufacturing operation and therapeutic process are complex. The number of randomized clinical trials (RCTs) investigating the effect of adjuvant vitamin A or K2 analog and lamivudine therapy is limited. Orally administered chemotherapy is a type of systemic treatment. As a convenient and non-invasive therapy, oral chemotherapeutic drug administration is easily adopted.

Although conventional systemic chemotherapy is well tolerated by inoperable HCC patients $(8,9), \mathrm{HCC}$ is widely considered to be chemotherapy-resistant, as the response rates are $\sim 20 \%$ for single-agent as well as combination chemotherapy (10). One of the most widely used chemotherapeutic agents is 5-fluorouracil. Several investigators have attempted to improve the survival of HCC patients postoperatively by conventional oral systemic chemotherapy (COSC) over the last few decades. However, these trials reported contradictory findings. Therefore, a systematic review is required to provide a more comprehensive analysis and assess the efficacy of COSC.

\section{Materials and methods}

Identification of trials. The Medline, Embase and Cochrane Library electronic databases were systematically searched through to April, 2012 and comparative studies investigating 
curative HR with and without COSC were identified using any of the following key words: hepatocellular carcinoma, hepatic tumor, liver tumor, postoperative, adjuvant, chemotherapy, or oral. The search was not limited to controlled or randomized trials for minimizing the chances of missing a study. Manual search of relevant references and review articles was also performed. There were no date and language restrictions. Only RCTs were included.

RCTs which assessed the effect of COSC for postoperative HCC were included in this review. Studies investigating liver metastases or postoperative recurrent $\mathrm{HCC}$ were excluded. The patients in the control group only received curative HR. The studies identified during the search were screened independently by two reviewers. Any disagreements were arbitrated by a third reviewer.

Types of outcome measures. The primary outcomes evaluated in this review were OS and recurrence rates. The secondary outcome was incidence of adverse events attributable to COSC.

Quality assessment. Two reviewers independently evaluated the quality of each retrieved trial in terms of randomization by sequence generation, allocation concealment, blinding of outcome assessors and reporting of intention-to-treat analysis. The trials were considered to be of low quality if they reported none of the items, of moderate quality if they reported on one or two items and of good quality if they reported on three or four items. The reporting of this systematic review is in accordance with the QUOROM statement (11).

Data extraction. Two reviewers independently extracted data regarding author details, methodological quality, number of patients, patient characteristics, interventions and outcomes. Discrepancies were resolved by consensus. When multiple publications of the same trial were identified, data were extracted from the multiple publications and reported as a single trial.

Statistical analysis. The data from each study were analyzed using the RevMan 5.1 software package. Odds ratios (ORs) with $95 \%$ confidence intervals (CIs) were calculated for OS and recurrence rate outcomes. Fixed- and random-effects models were applied in an 'intention-to-treat' analysis, i.e., all the patients were evaluated according to their group allocation. Patients whose endpoint was unknown were considered to be deceased or to have suffered tumor recurrence. Homogeneity between trials was analyzed using the $\chi^{2}$ test, with significance set at $\mathrm{P}>0.1$; the extent of heterogeneity was assessed by calculating $\mathrm{I}^{2}$. The point estimate of the OR was considered statistically significant at $\mathrm{P}<0.05$ if the $95 \%$ CI did not include the value 1 .

\section{Results}

Identification and characteristics of selected studies. From 916 citations identified by database searches, three eligible RCTs (12-14) involving a total of 286 patients were included in this systematic review (Fig. 1). One study was conducted in China (12) and two in Japan $(13,14)$. A definitive diagnosis of HCC was made based on histological evidence or a combina-

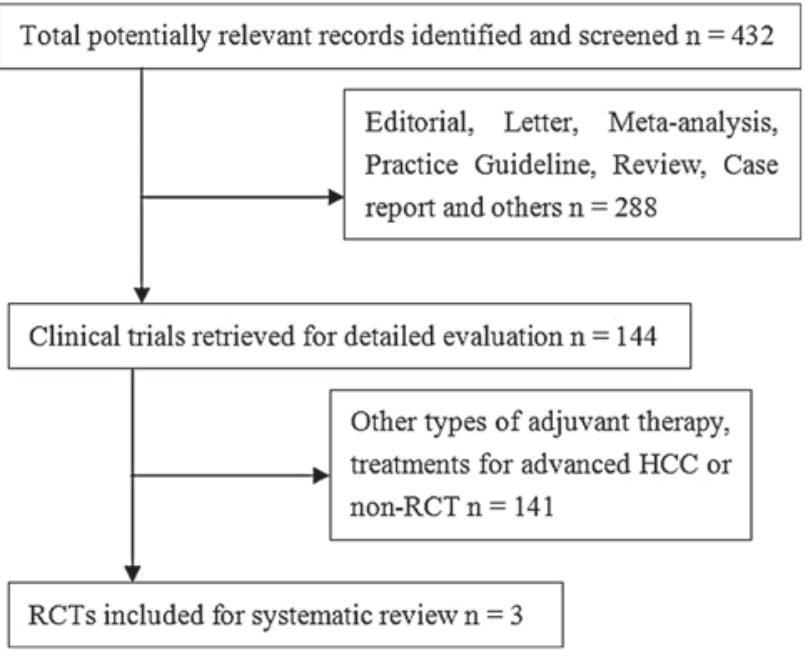

Figure 1. Flow chart of the study selection process. HCC, hepatocellular carcinoma; RCT, randomized clinical trial.

tion of several imaging modalities, e.g., hepatic angiography, enhanced computed tomography and magnetic resonance imaging. All the HCC patients underwent curative HR. No patient in the control group received any type of chemopreventive therapy prior to the discovery of recurrent HCC. The median follow-up was $>4$ years in all three RCTs (12-14). The patients in the treated group received oral capecitabine (12), uracil-tegafur (13), or carmofur (14), which are fluoropyrimidine drugs. Across the three studies, recurrence was measured and assessed in the same way by at least two imaging methods. The characteristics of the studies included in the review are summarized in Table I. In order to compare the efficacy of adjuvant COSC of other studies to the included studies, three other studies were systematically reviewed, including two RCTs $(15,16)$ and one case-control trial (17). All the HCC patients in these three trials underwent curative HR (15-17). The patients in the control group of two trials only received supportive care following HR $(15,17)$. By contrast, the patients in the treated group received adjuvant COSC, with or without other types of chemotherapy.

Quality of included RCTs. The risks of bias in the studies included in this systematic review are detailed in Table II. The methodological quality was considered to be high in the first (12), moderate in the second (13) and low in the third study (14).

Efficacy. Some of the efficacy of the included RCTs (12-14) and other trials (15-17) of adjuvant COSC for HCC patients are summarized in Table III.

OS. All the RCTs (12-14) described the OS. In the study by Xia et al (12), the median OS time was longer in the capecitabine group (60.0 vs. 52.5 months), but the difference was not statistically significant $(\mathrm{P}=0.22)$. The study by Yamamoto et al (14) concluded that the OS of patients with stage I disease was higher in the oral carmofur compared to that in the control group $(\mathrm{P}=0.08)$. However, in patients with stage II disease, no significant difference was observed $(\mathrm{P}=0.77)$. Interestingly, Hasegawa et al (13) drew an opposite conclusion, reporting 
Table I. Baseline characteristics of randomized clinical trials included into the systematic review.

\begin{tabular}{lcccccccc}
\hline & $\begin{array}{c}\text { Mean } \\
\text { follow-up, } \\
\text { years (range) }\end{array}$ & $\begin{array}{c}\text { Child-Pugh } \\
\text { classification } \\
\text { (A/B) }\end{array}$ & $\begin{array}{c}\text { Sample } \\
\text { size (n) }\end{array}$ & $\begin{array}{c}\text { Tumor size } \\
\text { (median, cm) }\end{array}$ & $\begin{array}{c}\text { Hepatitis (n) } \\
\text { (HBV/HCV) }\end{array}$ & $\begin{array}{c}\text { Cirrhosis } \\
(\%)\end{array}$ & $\begin{array}{c}\text { Vascular } \\
\text { invasion } \\
\text { (n) }\end{array}$ & (Refs.) \\
\hline Xia et al & $4.0(0.3-5.4)$ & $60 / 0$ & T:30 & 7.27 & $26 / \mathrm{NR}$ & 63.3 & 12 & $(12)$ \\
& & & $\mathrm{C}: 30$ & 6.34 & $24 / \mathrm{NR}$ & 70.0 & 10 & \\
Hasegawa et al & $4.8(0.5-7.9)$ & \multirow{2}{*}{$138 / 21$} & $\mathrm{~T}: 79$ & 3.3 & $14 / 58$ & 53.2 & 18 & $(13)$ \\
& & & $\mathrm{C}: 80$ & 3.4 & $15 / 56$ & 47.5 & 17 & \\
Yamamoto et al & $4-6$ & $\mathrm{NR}$ & $\mathrm{T}: 35$ & $\mathrm{NR}$ & $\mathrm{NR}$ & 100.0 & $\mathrm{NR}$ & $(14)$ \\
& & & $\mathrm{C}: 32$ & $\mathrm{NR}$ & $\mathrm{NR}$ & 100.0 & $\mathrm{NR}$ & \\
\hline
\end{tabular}

HBV, hepatitis B virus; HCV, hepatitis C virus; T, treated group; C, control group; NR, not reported.

Table II. Methodological quality assessment and internal validity of the included randomized clinical trials.

\begin{tabular}{lcccc}
\hline Study & $\begin{array}{c}\text { Random } \\
\text { allocation }\end{array}$ & $\begin{array}{c}\text { Concealment of } \\
\text { random allocation }\end{array}$ & $\begin{array}{c}\text { Blinding of persons } \\
\text { who assess treatment effects }\end{array}$ & $\begin{array}{c}\text { Intention-to-treat } \\
\text { analysis }\end{array}$ \\
\hline Xia et al & + & + & - & + \\
Hasegawa et al & + & - & - & + \\
Yamamoto et al & - & - & - & $(12)$ \\
\hline
\end{tabular}

Table III. Results of randomized clinical trials and other clinical trials of adjuvant conventional oral systemic chemotherapy for hepatocellular carcinoma.

\begin{tabular}{|c|c|c|c|c|c|c|}
\hline Study & $\begin{array}{l}\text { Drugs and dose } \\
\text { of the treated and control groups }\end{array}$ & Outcomes & $\begin{array}{l}\text { Treated } \\
\text { group }(\%)\end{array}$ & $\begin{array}{l}\text { Control } \\
\text { group }(\%)\end{array}$ & P-value & (Refs.) \\
\hline \multirow[t]{2}{*}{ Xia et al } & $\begin{array}{l}\text { Two weeks of capecitabine }\left(1,000 \mathrm{mg} / \mathrm{m}^{2}\right) \text { twice } \\
\text { a day, followed by } 1 \text { week of rest, for } 4-6 \text { cycles }\end{array}$ & 5-year DFS & 46.7 & 23.3 & $<0.05$ & \multirow[t]{2}{*}{$(12)$} \\
\hline & Supportive care & 5 -year OS & 62.5 & 39.8 & $>0.05$ & \\
\hline \multirow[t]{2}{*}{ Hasegawa et al } & Oral uracil-tegafur (300 mg/day) for 1 year & 5-year DFS & 29 & 29 & $>0.05$ & \multirow[t]{2}{*}{ (13) } \\
\hline & Supportive care & 5-year OS & 58 & 73 & $>0.05$ & \\
\hline \multirow[t]{2}{*}{ Yamamoto et al } & $\begin{array}{l}\text { Oral carmofur }(200 \mathrm{mg}) \text { twice daily for } \\
\text { as long as possible }\end{array}$ & 5-year DFS ${ }^{\mathrm{a}}$ & 50 & 19 & $<0.05$ & \multirow[t]{2}{*}{$(14)$} \\
\hline & Supportive care & 5 -year OS & 72 & 49 & $<0.05$ & \\
\hline \multirow[t]{2}{*}{ Kohno et al } & $\begin{array}{l}\text { Oral uracil-tegafur }(300 \mathrm{mg} / \text { day) for } 1 \text { year, } \\
\text { plus transarterial chemotherapy once } \\
\left.\text { (epirubicin } 40 \mathrm{mg} / \mathrm{m}^{2}\right)\end{array}$ & 5-year DFS & 17 & 14 & $>0.05$ & \multirow[t]{2}{*}{$(16)$} \\
\hline & Oral uracil-tegafur (300 mg/day) for 1 year & 5-year OS & 35 & 30 & $>0.05$ & \\
\hline \multirow[t]{2}{*}{ Ono et al } & $\begin{array}{l}\left.\text { Transarterial chemotherapy (epirubicin } 40 \mathrm{mg} / \mathrm{m}^{2}\right) \text {; } \\
\text { then intravenous chemotherapy (epirubicin } 40 \mathrm{mg} / \mathrm{m}^{2} \text { ), } \\
\text { once every } 3 \text { months for } 2 \text { years; in addition, } \\
\text { oral carmofur ( } 300 \mathrm{mg} / \text { day) for } 2 \text { years }\end{array}$ & 5-year DFS & 32 & 22.5 & $>0.05$ & \multirow[t]{2}{*}{$(15)$} \\
\hline & Supportive care & 5-year OS & 31.5 & 57 & $>0.05$ & \\
\hline \multirow[t]{2}{*}{ Takenaka et al } & $\begin{array}{l}\text { Oral uracil-tegafur or carmofur ( } 300-400 \mathrm{mg} / \mathrm{day}) \\
\text { for } 18 \text { months }\end{array}$ & 3-year DFS & 50 & 15 & $>0.05$ & \multirow[t]{2}{*}{$(17)$} \\
\hline & Supportive care & & & & & \\
\hline
\end{tabular}

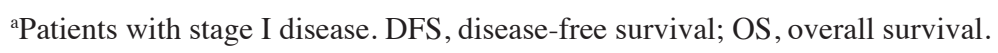




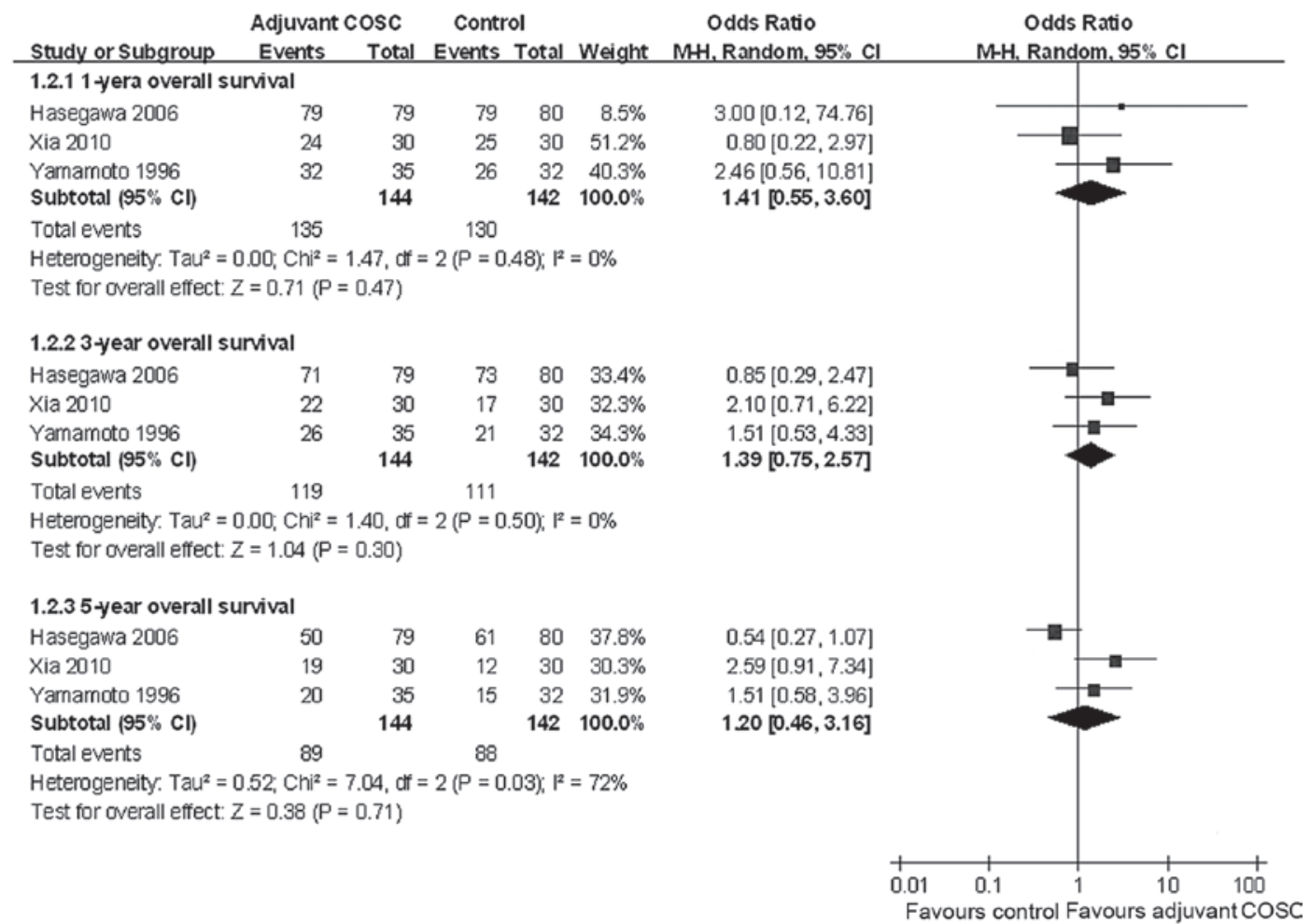

Figure 2. Meta-analysis of hepatocellular carcinoma patients' overall survival in randomized trials comparing hepatic resection plus conventional oral systemic chemotherapy (COSC) to hepatic resection alone. CI, confidence interval.

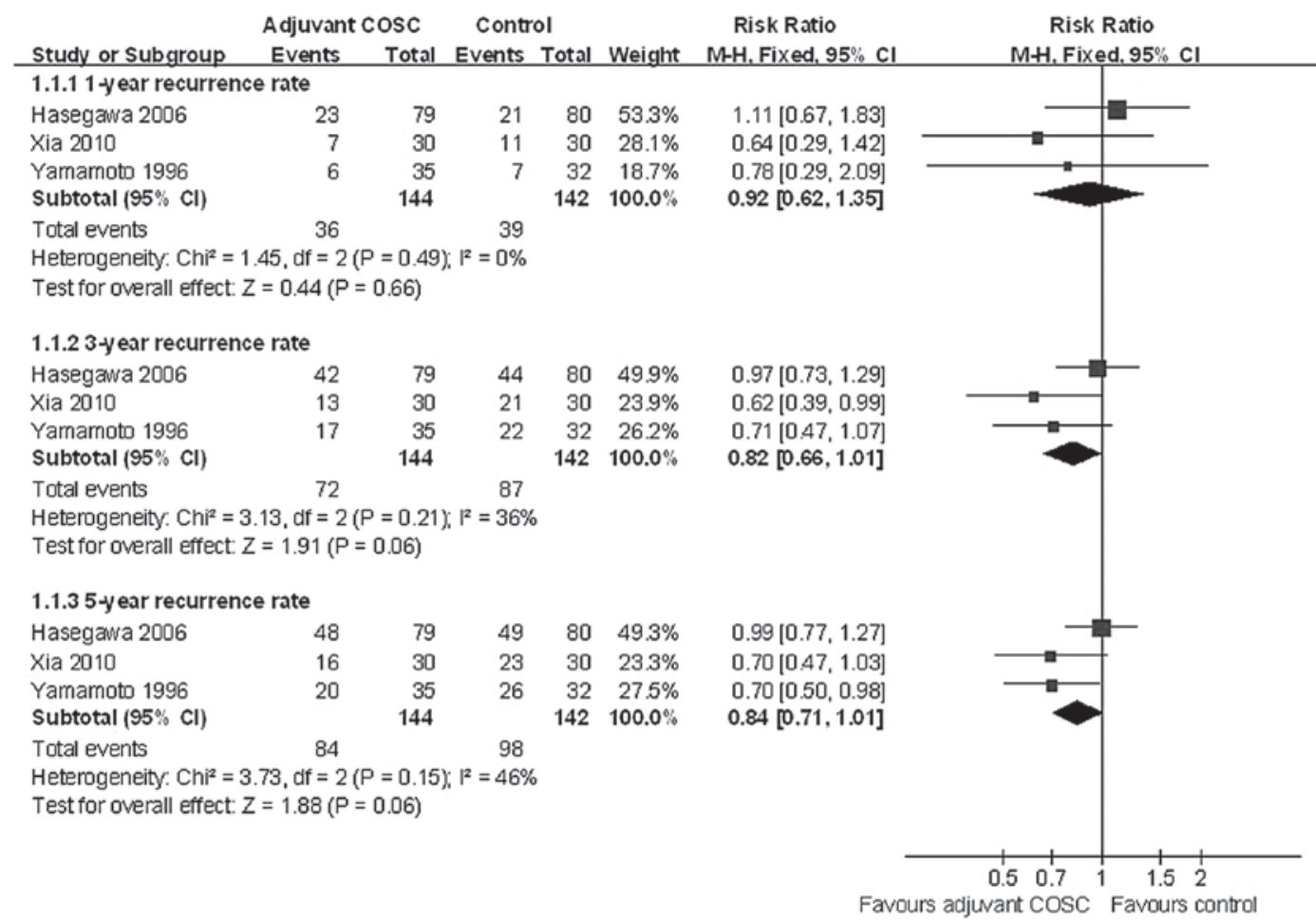

Figure 3. Meta-analysis of hepatocellular carcinoma recurrence in randomized trials comparing hepatic resection plus conventional oral systemic chemotherapy (COSC) to hepatic resection alone. CI, confidence interval.

that OS was marginally but not significantly worse in the uracil-tegafur compared to that in the control group $(\mathrm{P}=0.08)$.

A meta-analysis revealed that adjuvant COSC did not significantly increase the 1-, 3- and 5-year OS, with pooled ORs of 1.43
(95\% CI: 0.58-3.56, $\mathrm{P}=0.44), 1.39$ (95\% CI: 0.75-2.55, $\mathrm{P}=0.29)$ and 1.20 (95\% CI: 0.46-3.16, P=0.71), respectively (Fig. 2).

There were no significant differences between the two groups regarding long-term survival in the study by 
Kohno et al (16) $(\mathrm{P}=0.22)$ and the study by Ono et al $(15)$ $(\mathrm{P}=0.14)$.

Recurrence rates. All the three RCTs (12-14) reported recurrence rates. Compared to supportive care, capecitabine significantly decreased the recurrence rate $(\mathrm{P}=0.046)(12)$. Carmofur also improved the recurrence-free survival rates of patients with stage I disease $(\mathrm{P}=0.04)$. However, in patients with stage II disease, no significant difference was observed $(\mathrm{P}=1.00)$ (14). Yamamoto et al (14) concluded that the potential benefits of carmofur on tumor recurrence must be weighed against the risks of adverse reactions in patients with mild liver dysfunction. In the third study, however, the recurrence-free survival curves were similar between the groups $(\mathrm{P}=0.87)(13)$.

Although adjuvant COSC was expected to reduced recurrence, a meta-analysis did not revealed a statistically significant decrease in the incidence of the 1-, 3- and 5-year HCC recurrence rate, with pooled ORs of 0.92 (95\% CI: 0.62-1.35, $\mathrm{P}=0.66)$; 0.82 (95\% CI: 0.66-1.01, P=0.06); and 0.84 (95\% CI: 0.71-1.01, $\mathrm{P}=0.06$ ), respectively (Fig. 3).

All the three trials demonstrated no statistically significant difference in the disease-free survival (DFS) curves between the control and COSC with or without other type of chemotherapy groups (15-17).

Adverse effects of adjuvant COSC. There was no reported treatment-related mortality. In the study by Xia et al (12), the observed adverse reactions were generally mild. Nausea (23.3\%) and diarrhea (16.7\%) were the most common adverse effects associated with oral capecitabine. Two patients (7\%) withdrew from capecitabine therapy due to repeated grade III nausea or low white blood cell and platelet counts. Although treatment with uracil-tegafur was temporarily or permanently discontinued in $41 \%$ of the patients due to adverse effects, liver toxicity was negligible. Moreover, all the adverse events responded to conservative therapy (13). However, carmofur administration was suspended due to side effects in 9 of 21 patients $(42.9 \%)$ with clinical stage I and in 3 of 6 patients $(50 \%)$ with stage II cirrhosis, although the symptoms resolved within 2 months of suspension of the drug. The most common adverse effects were neuropathy (18.5\%) and liver dysfunction (18.5\%) (14).

\section{Discussion}

The aim of this systematic review was to assess the available evidence regarding the effect of HR plus COSC on OS and tumor recurrence in $\mathrm{HCC}$ patients. On the whole, adjuvant COSC with capecitabine, uracil-tegafur, or carmofur was well tolerated by the majority of the HCC patients. Two included RCTs indicated that adjuvant COSC significantly decreased the recurrence rate of $\mathrm{HCC}$ patients following curative HR $(12,14)$. However, the meta-analysis demonstrated that there was no statistically significant benefit regarding OS and tumor recurrence. Moreover, another two RCTs which investigated the efficacy of adjuvant COSC plus transarterial chemotherapy $(15,16)$ and one case-control trial $(17)$ did not demonstrate a statistically significant benefit of adjuvant COSC. These results were consistent with those of former studies evaluating the efficacy of $\operatorname{COSC}(9,18)$ and systemic intravenous chemotherapy for advanced HCC (19-22).
Conventional systemic therapy with a single agent or a combination of agents has provided marginal survival benefits.

Theoretically, adjuvant COSC may prevent metastatic recurrence caused by HCC cells present in the microcirculation, which were not identified by preoperative imaging, with fewer severe side effects on the liver. It is hypothesized that tumoricidal effects on lesions in the precancerous stage may be achieved by oral intake of anticancer agents, which induces a high portal drug concentration (14). Indeed, systemic chemotherapy is often difficult to perform on cirrhotic HCC patients following HR. First, the majority of the HCC patients are cirrhotic. Anticancer drugs may lead to further impairement of liver function. Hence, cirrhosis exerts a significant effect on the pharmacokinetics of systemic therapy for HCC. Second, certain drug resistance genes of HCC cells, such as P-glycoprotein, glutathione-S-transferase, heat shock proteins and mutations in p53, are overexpressed. Unlike other cancers, the majority of the reported deaths in HCC patients are attributed to liver disease rather than to HCC (13). In cirrhotic patients, adjuvant chemotherapy is associated with worse DFS and OS by negatively affecting liver function (14,23). Furthermore, accelerated repopulation of surviving tumor cells may occur after sequential chemotherapy (24). A marginally higher incidence of advanced recurrence in the adjuvant COSC group was reported by Hasegawa et al (13) and Lai et al (25) reported that adjuvant chemotherapy was associated with more frequent extrahepatic recurrences and a worse outcome. Therefore, systemic chemotherapy is not widely used. The majority of the HCC patients in this systematic review were infected by hepatitis $\mathrm{B} / \mathrm{C}$ virus and/or cirrhotic. The meta-analysis demonstrated that adjuvant COSC was likely to decrease the recurrence rate and improve the OS. However, no statistically significant benefit was observed.

Certain double-blind, multicenter, phase III RCTs indicated that oral sorafenib is effective for the treatment of advanced HCC (26-28). A phase III trial is ongoing to evaluate the safety and efficacy of adjuvant sorafenib compared to placebo in the treatment of HCC. Oral sorafenib may prove to be a viable option for the treatment of HCC following curative HR.

A major drawback of this review is that the number of the included patients, who were all Asian, was relatively small. Although we systematically searched Medline, Embase and the Cochrane Library databases and reviewed two RCTs and one case-control trial investigating adjuvant systemic chemotherapy for HCC, there is a risk of random errors. Furthermore, some of the important patient characteristics, such as tumor size and hepatitis status, were not described. In addition, the randomization procedure and allocation concealment remained unclear in certain studies. Due to these limitations, the results and the conclusions of the present study should be interpreted with caution.

In summary, although adjuvant COSC appears to be well tolerated, our results demonstrated only marginal benefits for HCC patients undergoing curative HR. Further trials should be conducted to investigate novel adjuvant therapies, such as treatment with multikinase inhibitors.

\section{Acknowledgements}

This study was supported by the Self-Raised Scientific Research Fund of the Ministry of Health of Guangxi Province 
(nos. Z2012345 and Z2014241) and Youth Science Foundation of Guangxi Medical University (no. GXMUYSF201302) to Z.J.H., and by the Guangxi Natural Science Foundation (no. 2011GXNSFD 018032) to L.L.Q.

\section{References}

1. Imamura H, Matsuyama Y, Tanaka E, et al: Risk factors contributing to early and late phase intrahepatic recurrence of hepatocellular carcinoma after hepatectomy. J Hepatol 38 : 200-207, 2003

2. Zhong C, Guo RP, Li JQ, et al: A randomized controlled trial of hepatectomy with adjuvant transcatheter arterial chemoembolization versus hepatectomy alone for stage III A hepatocellular carcinoma. J Cancer Res Clin Oncol 135: 1437-1445, 2009.

3. Capussotti L, Muratore A, Amisano M, et al: Liver resection for hepatocellular carcinoma on cirrhosis: analysis of mortality, morbidity and survival - a European single center experience. Eur J Surg Oncol 31: 986-993, 2005.

4. Lang H, Sotiropoulos GC, Brokalaki EI, et al: Survival and recurrence rates after resection for hepatocellular carcinoma in noncirrhotic livers. J Am Coll Surg 205: 27-36, 2007.

5. Zhong JH, Li H, Li LQ, et al: Adjuvant therapy options following curative treatment of hepatocellular carcinoma: a systematic review of randomized trials. Eur J Surg Oncol 38: 286-295, 2012.

6. Zhong JH and Li LQ: Postoperative adjuvant transarterial chemoembolization for participants with hepatocellular carcinoma: a meta-analysis. Hepatol Res 40: 943-953, 2010.

7. Zhong JH, Ma L, Wu LC, et al: Adoptive immunotherapy for postoperative hepatocellular carcinoma: a systematic review. Int J Clin Pract 66: 21-27, 2012.

8. Llovet JM, Ruff P, Tassopoulos N, et al: A phase II trial of oral eniluracil/5-fluorouracil in patients with inoperable hepatocellular carcinoma. Eur J Cancer 37: 1352-1358, 2001.

9. Benson AB III, Mitchell E, Abramson N, Klencke B, Ritch P, Burnhan JP, McGuirt C, Bonny T, Levin J and Hohneker J: Oral eniluracil/5-fluorouracil in patients with inoperable hepatocellular carcinoma. Ann Oncol 13: 576-581, 2002.

10. Johnson PJ: Hepatocellular carcinoma: is current therapy really altering outcome? Gut 51: 459-462, 2002.

11. Moher D, Cook DJ, Eastwood S, et al: Improving the quality of reports of meta-analyses of randomised controlled trials: the QUOROM statement. QUOROM Group. Br J Surg 87: 1448-1454, 2000.

12. Xia Y, Qiu Y, Li J, et al: Adjuvant therapy with capecitabine postpones recurrence of hepatocellular carcinoma after curative resection: a randomized controlled trial. Ann Surg Oncol 17: 3137-3144, 2010.

13. Hasegawa K, Takayama T, Ijichi M, et al: Uracil-tegafur as an adjuvant for hepatocellular carcinoma: a randomized trial. Hepatology 44: 891-895, 2006.
14. Yamamoto M, Arii S, Sugahara K and Tobe T: Adjuvant oral chemotherapy to prevent recurrence after curative resection for hepatocellular carcinoma. Br J Surg 83: 336-340, 1996.

15. Ono T, Nagasue N, Kohno H, et al: Adjuvant chemotherapy with epirubicin and carmofur after radical resection of hepatocellular carcinoma: a prospective randomized study. Semin Oncol 24 (Suppl 6): 18-25, 1997.

16. Kohno H, Nagasue N, Hayashi T, et al: Postoperative adjuvant chemotherapy after radical hepatic resection for hepatocellular carcinoma (HCC). Hepatogastroenterology 43: 1405-1409, 1996.

17. Takenaka K, Yoshida K, Nishizaki T, et al: Postoperative prophylactic lipiodolization reduces the intrahepatic recurrence of hepatocellular carcinoma. Am J Surg 169: 400-405, 1995.

18. Patt YZ, Hassan MM, Aguayo A, et al: Oral capecitabine for the treatment of hepatocellular carcinoma, cholangiocarcinoma, and gallbladder carcinoma. Cancer 101: 578-586, 2004.

19. Edeline J, Raoul JL, Vauleon E, et al: Systemic chemotherapy for hepatocellular carcinoma in non-cirrhotic liver: a retrospective study. World J Gastroenterol 15: 713-716, 2009.

20. Zhu AX: Systemic treatment of hepatocellular carcinoma: dawn of a new era? Ann Surg Oncol 17: 1247-1256, 2010.

21. Uchino K, Obi S, Tateishi R, et al: Systemic combination therapy of intravenous continuous 5-fluorouracil and subcutaneous pegylated interferon alfa-2a for advanced hepatocellular carcinoma. J Gastroenterol 47: 1152-1159, 2012.

22. Cao H, Phan H and Yang LX: Improved chemotherapy for hepatocellular carcinoma. Anticancer Res 32: 1379-1386, 2012.

23. Ono T, Yamanoi A, Nazmy El Assal O, et al: Adjuvant chemotherapy after resection of hepatocellular carcinoma causes deterioration of long-term prognosis in cirrhotic patients: metaanalysis of three randomized controlled trials. Cancer 91: 2378-2385, 2001.

24. Davis AJ and Tannock JF: Repopulation of tumour cells between cycles of chemotherapy: a neglected factor. Lancet Oncol 1: 86-93, 2000.

25. Lai EC, Lo CM, Fan ST, et al: Postoperative adjuvant chemotherapy after curative resection of hepatocellular carcinoma: a randomized controlled trial. Arch Surg 133: 183-188, 1998.

26. Abou-Alfa GK, Johnson P, Knox JJ, et al: Doxorubicin plus sorafenib vs. doxorubicin alone in patients with advanced hepatocellular carcinoma: a randomized trial. JAMA 304: 2154-2160, 2010.

27. Llovet JM, Ricci S, Mazzaferro V, et al: Sorafenib in advanced hepatocellular carcinoma. N Engl J Med 359: 378-390, 2008.

28. Cheng AL, Kang YK, Chen Z, et al: Efficacy and safety of sorafenib in patients in the Asia-Pacific region with advanced hepatocellular carcinoma: a phase III randomised, double-blind, placebo-controlled trial. Lancet Oncol 10: 25-34, 2009. 